\title{
Measurement of proton-distribution radii of neutron-rich nitrogen isotopes
}

Soumya Bagchi ${ }^{1,2,3, *}$, Rituparna Kanungo ${ }^{1,4}$, Wataru Horiuchi ${ }^{5}$, Gaute Hagen ${ }^{6,7}$, Titus D. Morris ${ }^{6,7}$, Steven Ragnar Stroberg $^{4}$, Toshio Suzuki ${ }^{8}$, Frederic Ameil ${ }^{2}$, Joel Atkinson ${ }^{1}$, Yassid Ayyad ${ }^{9}$, Dolores Cortina-Gil ${ }^{9}$, Iris Dillmann $^{2}$, Alfredo Estradé $^{1,2}$, Alexey Evdokimov ${ }^{2}$, Fabio Farinon ${ }^{2}$, Hans Geissel ${ }^{2,3}$, Giulia Guastalla ${ }^{2}$, Rudolf Janik ${ }^{10}$, Satbir Kaur ${ }^{1,11}$, Ronja Knöbel $^{2}$, Jan Kurcewicz ${ }^{2}$, Yury Litvinov ${ }^{2}$, Michele Marta ${ }^{2}$, Magdalena Mostazo ${ }^{9}$, Ivan Mukha ${ }^{2}$, Chiara Nociforo ${ }^{2}$, Hooi Jin Ong ${ }^{12}$, Stephane Pietri ${ }^{2}$, Andrej Prochazka ${ }^{2}$, Christoph Scheidenberger ${ }^{2,3}$, Branislav Sitar ${ }^{10}$, Peter Strmen ${ }^{10}$, Maya Takechi $^{2}$, Junki Tanaka ${ }^{12}$, Yoshiki Tanaka ${ }^{1,2,3}$, Isao Tanihata ${ }^{12,13}$, Satoru Terashima ${ }^{13}$, Jossitt Vargas ${ }^{9}$, Helmut Weick ${ }^{2}$, and John Stuart Winfield ${ }^{2}$

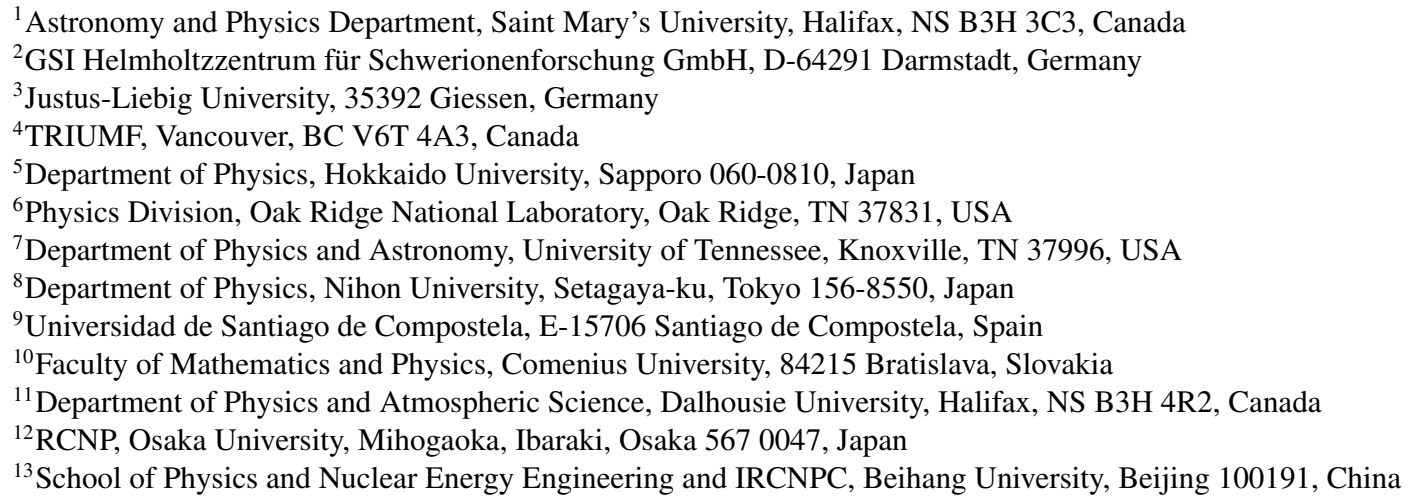

\begin{abstract}
Measurement of root-mean-square radii of proton distributions of ${ }^{17-22} \mathrm{~N}$ from charge-changing cross section shows the emergence of thick neutron skin towards the neutron-drip line. Signature of $N=14$ shell gap has been found in nitrogen isotopes along with the emergence of neutron halo in ${ }^{22} \mathrm{~N}$. The measured radii are in good agreement with the shell model calculations.
\end{abstract}

\section{Introduction}

With large neutron-to-proton ratios far from the valley of stability, nuclei develop exotic structures such as neutron skin, nuclear halo, modifications of conventional shells and apperance of new shell gaps. Evidence for breaking down of $N=8$ shell gap has been found in ${ }^{11} \mathrm{Li}$ and ${ }^{11} \mathrm{Be}[1-3]$ while a new shell gap at the neutron number $N=16$ has been found in carbon, nitrogen and oxygen isotopes at the drip line [4]. Another sub-shell gap at $N=14$ has been found in oxygen and is predicted to disappear in carbon [5]. Since nitrogen is in the transitional region between oxygen and carbon, it is interesting to investigate the presence of the $N=14$ shell gap in nitrogen. Systematic studies of root-mean-square radii of proton-distribution along an isotopic chain can shed light on nuclear models as well as on the shell evolution [6].

\section{Experimental setup}

In this work, the determination of root-mean-square radii of proton-distribution (protons treated as point-like particles and therefore referred further as point-proton radii)

*e-mail: s.bagchi@gsi.de from the measured charge-changing cross sections $\left(\sigma_{C C}\right)$ of neutron-rich isotopes ${ }^{17-22} \mathrm{~N}$ and stable isotopes ${ }^{14,15} \mathrm{~N}$ is presented. The experiment was performed at the fragment separator FRS at GSI. Beams of ${ }^{14,15} \mathrm{~N}$ and ${ }^{17-22} \mathrm{~N}$ were produced by fragmentation of ${ }^{22} \mathrm{Ne}$ and ${ }^{40} \mathrm{Ar}$, respectively, at $1 \mathrm{~A} \mathrm{GeV}$, impinging on a $6.3 \mathrm{~g} / \mathrm{cm}^{2}$ thick Be target. The isotopes of interest were separated in flight and identified using their magnetic rigidity $(B \rho)$, time-of-flight (ToF) between the focal planes (F2-F4) of the FRS, and the energy-loss $(\Delta E)$ measured in a multi-sampling ionization chamber (MUSIC) placed at the final focal plane F4. The $\sigma_{C C}$ was measured with a $4.010 \mathrm{~g} / \mathrm{cm}^{2}$ thick carbon target placed at F4. The experimental setup is discussed in more details in Ref. [7].

To obtain $\sigma_{C C}$, the number $\left(N_{i n}\right)$ of incident nuclei of interest ${ }^{A} \mathrm{Z}$ is determined from event-by-event counting. After the reaction target, the number of nuclei with the same charge $\mathrm{Z}$ as the incident nuclei $\left(N_{\text {SameZ }}\right)$ are identified using the MUSIC detector. The $\sigma_{C C}$ is then obtained from the relation $\sigma_{C C}=t^{-1} \ln \left(R_{T_{\text {out }}} / R_{T_{\text {in }}}\right)$. Here $R_{T_{\text {in }}}$ and $R_{T_{\text {out }}}$ are the ratios of $N_{\text {SameZ }} / N_{\text {in }}$ with and without the reaction target, respectively, and $t$ is the target thickness. The term $R_{T_{\text {out }}}$ attributes for losses due to interactions with non-target materials. Phase-space restriction of the inci- 
dent beam at the target is required to eliminate events having large incident angles and positions far from the center. Details of the measurement procedure can be found in Ref. [7].

\section{Results and discussion}

To obtain the proton-distribution radii from the measured cross section $\sigma_{C C}$, finite-range Glauber model framework is used [8]. For stable isotopes (e.g. ${ }^{10} \mathrm{~B},{ }^{12-14} \mathrm{C},{ }^{14} \mathrm{~N}$ ), the obtained proton-distribution radii are in good agreement with the electron-scattering data $[7,9,10]$. The extracted point-proton-distribution radii $\left(R_{p}\right)$ from $\sigma_{C C}$ are shown in Fig. 1.

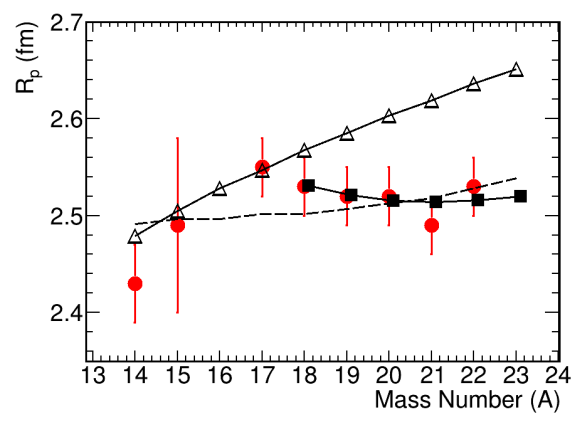

Figure 1. The experimental point-proton radii are shown with red filled circles. Shell model predictions using the YSOX interaction and WS (HO) potentials are shown with black filled squares (black open triangles). RMF theory predictions are given with the black dashed curve from Ref. [11].

The decrease of $R_{p}$ from ${ }^{17} \mathrm{~N}$ to ${ }^{21} \mathrm{~N}$ within the uncertainties suggests the reduction in deformation and the transition towards sphericity at the $N=14$ shell closure. An increase of $R_{p}$ has been observed within uncertainties from ${ }^{21} \mathrm{~N}$ to ${ }^{22} \mathrm{~N}$. In ${ }^{22} \mathrm{~N}$, the valence neutron is in the $2 s_{1 / 2}$ orbital with a closed-shell core of ${ }^{21} \mathrm{~N}$. As a consequence, the center-of-mass (c.m.) of ${ }^{22} \mathrm{~N}$ is different from that of the ${ }^{21} \mathrm{~N}$ core which leads to the c.m. motion and hence it smears the core density, resulting in a larger core size [7]. Similar phenomenon has been observed in the two neutron halo ${ }^{11} \mathrm{Li}[12]$ and ${ }^{6} \mathrm{He}$ [13] isotopes.

Shell model calculations are compared with the experimental data and are shown in Fig. 1. Both harmonic oscillator (HO) and Woods-Saxon (WS) wave functions are used in the YSOX hamiltonian. The combination of both
$\mathrm{HO}$ and WS wavefunctions depicts that the proton radii of the nitrogen isotopes increase from ${ }^{13} \mathrm{~N}$ to ${ }^{17} \mathrm{~N}$ and then a decrease is seen with a shallow minimum at the neutron number $N=14$. In Fig. 1, the relativistic mean field (RMF) predictions [11] are shown which are consistent with the experimental data. Ab initio, in-medium similarity re-normalization group, calculations with a stateof-the-art chiral nucleon-nucleon and three-nucleon interaction reproduce well the data towards the neutron dripline isotopes rather than the whole isotopic trend of the radii [7].

The matter-distribution radii can be obtained from the measured interaction cross sections [14] using the finiterange Glauber model framework. Together with the information on the matter-distribution and proton-distribution radii, thick neutron skins for ${ }^{19-21} \mathrm{~N}$, have been found, while for ${ }^{22} \mathrm{~N}$ a neutron halo-like structure develops [7].

For nitrogen isotopes, the proton-neutron tensor interaction is attractive for $\mathrm{p}\left(1 p_{1 / 2}\right)-\mathrm{n}\left(1 d_{5 / 2}\right)$ orbitals, thereby reducing the gap between proton $1 p_{1 / 2}$ and $1 p_{3 / 2}$ orbitals when more neutrons are added to the $1 d_{5 / 2}$ orbital, resulting in a small point proton radius in ${ }^{21} \mathrm{~N}$. This attractive interaction also lowers the neutron $1 d_{5 / 2}$ orbital leading to the $N=14$ shell gap [7] in neutron-rich nitrogen isotopes.

\section{References}

[1] A. Navin, et al., Phys. Rev. Lett. 85, 266 (2009).

[2] H. Iwasaki, et al., Phys. Lett. B 491, 8-14 (2000).

[3] H. Simon, et al., Phys. Rev. Lett. 83, 496 (1999).

[4] Z. Dlouhý, J. Mrázek and D. Baiborodin, AIP Conf. Proc. 610, 736 (2002).

[5] M.J. Strongman, et al., Phys. Rev. C 80, 021302(R) (2009).

[6] I. Angeli, K.P. Marinova, At. Data Nucl. Data Tables 99, 69 (2013).

[7] S. Bagchi, et al., Phys. Lett. B 790, 251-256 (2019).

[8] Y. Suzuki, et al., Phys. Rev. C 94, 011602(R) (2016).

[9] A. Estradé, et al., Phys. Rev. Lett. 113, 132501 (2014).

[10] R. Kanungo, et al., Phys. Rev. Lett. 117, 102501 (2016).

[11] J. Meng, et al., Phys. Lett. B 532, 209-214 (2002).

[12] R. Sánchez, et al., Phys. Rev. Lett. 96, 033002 (2006).

[13] P. Mueller, et al., Phys. Rev. Lett. 99, 252501 (2007).

[14] A. Ozawa, et al., Nucl. Phys. A 693, 32-62 (2001). 